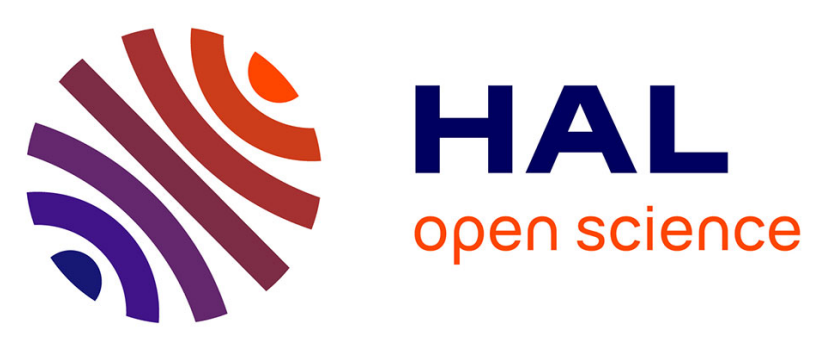

\title{
Uncertainty quantification of voice signal production mechanical model and experimental updating
}

Edson Cataldo, Christian Soize, Rubens Sampaio

\section{To cite this version:}

Edson Cataldo, Christian Soize, Rubens Sampaio. Uncertainty quantification of voice signal production mechanical model and experimental updating. Mechanical Systems and Signal Processing, 2013, 40 (2), pp.718-726. 10.1016/j.ymssp.2013.06.036 . hal-00839777

\section{HAL Id: hal-00839777 \\ https://hal.science/hal-00839777}

Submitted on 22 Jul 2013

HAL is a multi-disciplinary open access archive for the deposit and dissemination of scientific research documents, whether they are published or not. The documents may come from teaching and research institutions in France or abroad, or from public or private research centers.
L'archive ouverte pluridisciplinaire HAL, est destinée au dépôt et à la diffusion de documents scientifiques de niveau recherche, publiés ou non, émanant des établissements d'enseignement et de recherche français ou étrangers, des laboratoires publics ou privés. 


\title{
Uncertainty quantification of voice signal production mechanical model and experimental updating
}

\author{
E. Cataldo ${ }^{\mathrm{a}}$, C. Soize ${ }^{\mathrm{b}}$, R. Sampaio ${ }^{\mathrm{c}}$ \\ ${ }^{a}$ Universidade Federal Fluminense, Applied Mathematics Departament and Graduate \\ program in Telecommunications Engineering, Rua Mário Santos Braga, S/N, Centro, \\ Niteroi, RJ, CEP: 24020-140, Brazil \\ ${ }^{b}$ Université Paris-Est, Laboratoire Modelisation et Simulation Multi Echelle, MSME UMR \\ 8208 CNRS, 5 Bd Descartes, 77454 Marne-La-Vallée, France \\ ${ }^{c}$ PUC-Rio, Mechanical Engineering Departament, Rua Marquês de São Vicente, 225, \\ Gavea, Rio de Janeiro, RJ, CEP: 22453-900, Brazil
}

\begin{abstract}
The aim of this paper is to analyze the uncertainty quantification in a voice production mechanical model and update the probability density function corresponding to the tension parameter using the Bayes method and experimental data. Three parameters are considered uncertain in the voice production mechanical model used: the tension parameter, the neutral glottal area and the subglottal pressure. The tension parameter of the vocal folds is mainly responsible for the changing of the fundamental frequency of a voice signal, generated by a mechanical/mathematical model for producing voiced sounds. The three uncertain parameters are modeled by random variables. The probability density function related to the tension parameter is considered uniform and the probability density functions related to the neutral glottal area and the subglottal pressure are constructed using the Maximum Entropy Principle. The output of the stochastic computational model is the random voice signal and the Monte Carlo method is used to solve the stochastic equations allowing realizations of the random voice signals to be generated. For each realization of the random voice signal, the corresponding realization of the random fundamental frequency is calculated and the prior pdf of this random fundamental frequency is then estimated. Experimental data are available for the fundamental frequency and the posterior probability density function of the random tension parameter is then estimated using the Bayes method. In addition, an application is performed considering a case with a pathology in the vocal folds. The strategy developed here is important mainly due to two things. The first one is related to the possibility of updating the probability density function of a parameter, the tension parameter of the vocal folds, which cannot be measured direct and the second one is related to the construction of the likelihood function. In gen-
\end{abstract}

Email addresses: ecataldo@im.uff.br (E. Cataldo), christian.soize@univ-paris-est.fr (C. Soize), rsampaio@puc-rio.br (R. Sampaio) 
eral, it is predefined using known pdf. Here, it is constructed in a new and different manner, using the own system considered.

Key words: Uncertainty quantification; Voice production; Probabilistic model; Bayes method

The production of voiced sounds (vowels are particular cases of voiced sounds) starts with the contraction-expansion of the lungs causing an airflow (due to the difference of pressure between the lungs and the mouth), which will induce the auto-oscillation of the vocal folds (located in the larynx). After passing through the glottis and due to the movement of the vocal folds, the airflow is transformed into pulses of air which are generated (quasi)-periodically. The pressure signal created is so called the glottal signal, which will further be filtered and amplified by the vocal tract to generate the sound we hear. The fundamental frequency of the voice signal, which is the frequency of the vocal folds oscillation, is the inverse of the period of the glottal signal. As the glottal signal is not exactly periodic, for each time interval corresponding to a complete cycle of the vocal folds, a different fundamental frequency is associated with. This variation of the fundamental frequency in a voice signal is known in the literature as jitter (see for instance Titze, 1994). So, the voice signals constitute a stochastic process and the fundamental frequency will be a random variable.

Some authors have modeled the vocal folds dynamics, mainly in a deterministic way (Koizumi et al., 1976; Lous et al., 1998; Zhang et al., 2005). One of these models is the well-known model proposed by Ishizaka and Flanagan (1972) and it will be used here because it has provided a simple and effective representation of the system for studying the underlying dynamics of voice production.

In the present work, the Ishizaka and Flanagan model is used as the voice production mechanical model. The three main parameters of this model are the tension parameter, the neutral glottal area and the subglottal pressure. It should be noted that the tension parameter of the vocal folds is mainly responsible for the changing of the fundamental frequency of a voice signal, generated by a mechanical/mathematical model for producing voiced sounds. These three parameters are considered as uncertain parameters. The propagation of uncertainties in the voice production mechanical model is carried out using the Monte Carlo method. For each realization of the random voice signal, the corresponding realization of the random fundamental frequency is calculated and the prior pdf of this random fundamental frequency is then estimated. Two experimental validations have been presented using experimental data available for the fundamental frequency, one considering a normal voice and another considering a case with pathology in the vocal folds. The posterior probability density function of the random tension parameter, which cannot be easily measured, is estimated using the Bayes method. The likelihood function is construction using the own 
model considered for producing voice.

\section{Brief description of the Ishizaka and Flanagan model}

A diagram of the model is shown in Fig 1. The dynamics of the system is

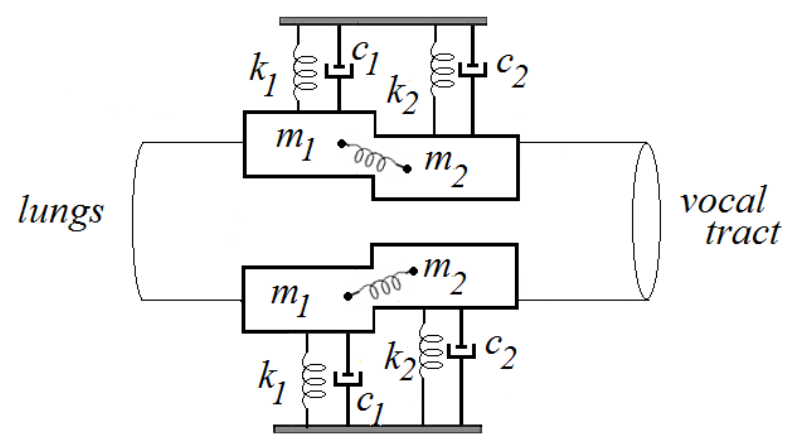

Figure 1: Two-mass model of the vocal folds.

given by Eqs. (1) and (2) (Cataldo et. al., 2008, 2009):

$$
\begin{aligned}
& \psi_{1}(\mathbf{w}) \dot{u}_{g}+\psi_{2}(\mathbf{w})\left|u_{g}\right| u_{g}+\psi_{3}(\mathbf{w}) u_{g}+\frac{1}{\tilde{c}_{1}} \int_{0}^{t}\left(u_{g}(\tau)-u_{1}(\tau)\right) d \tau-y=0 \\
& {[M] \ddot{\mathbf{w}}+[C] \dot{\mathbf{w}}+[K] \mathbf{w}+\mathbf{h}\left(\mathbf{w}, \dot{\mathbf{w}}, u_{g}, \dot{u}_{g}\right)=0}
\end{aligned}
$$

where $\mathbf{w}(t)=\left(x_{1}(t), x_{2}(t), u_{1}(t), u_{2}(t), u_{r}(t)\right)$, the functions $x_{1}$ and $x_{2}$ are the displacements of the masses, $u_{1}$ and $u_{2}$ describe the air volume flow through the (two) tubes that model the vocal tract and $u_{r}$ is the air volume flow through the mouth. The subglottal pressure is denoted by $y$ and $u_{g}$ is the function that represent the glottal pulses signal. The output radiated pressure function $p_{r}$ is given by $p_{r}(t)=u_{r}(t) r_{r}$, in which $r_{r}=\frac{128 \rho v_{c}}{9 \pi^{3} y_{2}^{2}}, \rho$ is the air density, $v_{c}$ is the sound velocity, and $y_{2}$ is the radius of the second tube. The description of the other quantities that appear in the equation and a detailed discussion of the model, including its implementation, can be found in (Cataldo et al., 2009).

The process to generate a voiced sound is complex and its modeling involves a lot of quantities which should be controlled. Here, the interest is in the changing of the fundamental frequency. The three main parameters responsible for these changings, as discussed in (Ishizaka and Flanagan, 1972; Cataldo et al., 2008, 2009) are described in the following:

$a_{g 0}$ : the area at rest between the vocal folds, called the neutral glottal area.

$y$ : the subglottal pressure. 
$q$ : the tension parameter which controls the fundamental frequency of the vocal-fold vibrations because vocal fold abduction and tension are the main factors used by a speaker to control phonation. In order to control the fundamental frequency of the vocal folds, parameters $m_{1}, k_{1}, m_{2}, k_{2}$, $k_{c}$ are written as $m_{1}=\widehat{m}_{1} / q, k_{1}=q \widehat{k}_{1}, m_{2}=\widehat{m}_{2} / q, k_{2}=q \widehat{k}_{2}, k_{c}=q \widehat{k}_{c}$, in which $\widehat{m}_{1}, \widehat{k}_{1}, \widehat{m}_{2}, \widehat{k}_{2}, \widehat{k}_{c}$ are fixed values.

These three parameters are considered as uncertain and are then modeled by random variables. Consequently, the output of the model is a random voice signal which is then a stochastic process. It means that for each realization of these three random variables, a realization of the random voice signal is produced.

Figures 2 and 3 show the blocks diagram with the deterministic system and the corresponding stochastic system.

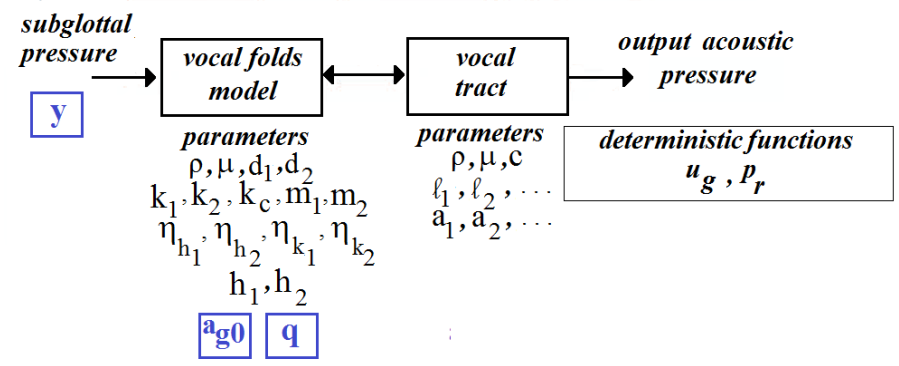

Figure 2: Deterministic system.

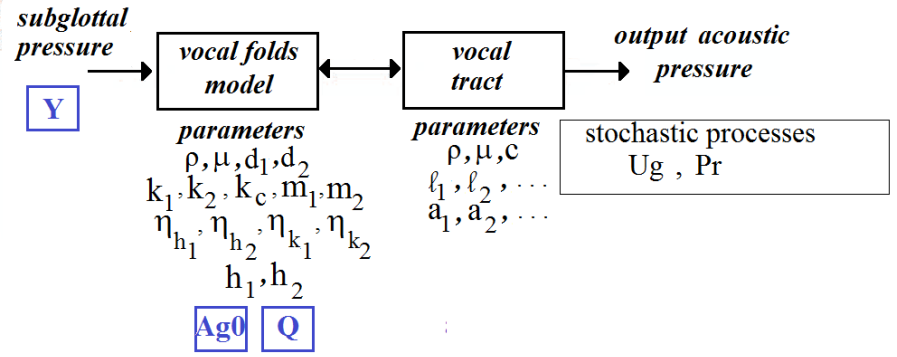

Figure 3: Corresponding stochastic system.

The prior probability density functions of these random variables which model the uncertain parameters will be constructed by using the Maximum Entropy Principle (Jaynes, 1957a, 1957b) which consists in maximizing the entropy (Shannon, 1948) from Information Theory, under the constraints defined 
by the available information. The entropy measures the level of uncertainties and is written as

$$
S\left(p_{X}\right)=-\int_{-\infty}^{+\infty} p_{X}(x) \ln \left(p_{X}(x)\right) d x
$$

in which $p_{X}$ is the pdf of the random variable $X$. The constraints defined by the available information are written as

$$
\int_{-\infty}^{+\infty} p_{X}(x) d x=1 \text { and } \int_{-\infty}^{+\infty} p_{X}(x) g_{i}(x) d x=a_{i}, i=1, \ldots, m,
$$

in which $a_{i}^{\prime} s$ are usable information related to the functions $g_{i}^{\prime} s$. According to the first part of the principle, only probability distributions consistent with the constraints given should be used. However, an infinity of probability distributions compatible with the constraints may exist. The second part of the principle states the way to choose one among the many pdf's that satisfy the constraints, the (unique) pdf that maximizes the entropy.

\section{Prior probabilistic model of the uncertain parameters}

The three parameters $q, a_{g 0}$ and $y$ are modeled by the random variables $Q, A_{g 0}$ and $Y$, respectively. Consequently, parameters $m_{1}, k_{1}, m_{2}, k_{2}$, and $k_{c}$ become random variables denoted by $M_{1}, K_{1}, M_{2}, K_{2}$, and $K_{c}$ given by $M_{1}=\widehat{m}_{1} / Q, K_{1}=Q \widehat{k}_{1}, M_{2}=\widehat{m}_{2} / Q, K_{2}=Q \widehat{k}_{2}$, and $K_{c}=Q \widehat{k}_{c}$. Since no information is available concerning cross statistical moments between random variables $Q, A_{g 0}$ and $Y$, they will be considered independent. Taking into account the sensitivity of the fundamental frequency with respect to $Q$ (Ishizaka and Flanagan, 1972; Cataldo et al., 2009), we propose to construct a Bayesian posterior for the pdf of $Q$ using experimental data. In such a case the prior pdf of $Q$ is chosen as a uniform pdf on an adapted interval $[a, b]$ and is then written as

$$
p_{Q}^{\text {prior }}(q)=\mathbf{1}_{[a, b]}(q) \frac{1}{b-a} .
$$

Concerning the construction of the prior pdf of random variables $A_{g 0}$ and $Y$, we use the Maximum Entropy principle. The details about the construction of the pdf's related to these two random variables can be found in (Cataldo et al., 2009) and their expressions are recalled hereinafter. The pdf for $A_{g 0}$ is written as

$$
p_{A_{g 0}}\left(a_{g 0}\right)=\mathbf{1}_{] 0,+\infty}\left[e^{-\lambda_{0}-\lambda_{1} a_{g_{0}}-\lambda_{2}\left(a_{g_{0}}\right)^{2}},\right.
$$

where $\lambda_{0}, \lambda_{1}$ and $\lambda_{2}$ are the solution of the three following equations,

$$
\int_{-\infty}^{+\infty} p_{A_{g 0}}\left(a_{g 0}\right) d a_{g 0}=1
$$




$$
\begin{aligned}
& \int_{-\infty}^{+\infty} a_{g 0} p_{A_{g 0}}\left(a_{g 0}\right) d a_{g 0}=\underline{A}_{g 0}, \\
& \int_{-\infty}^{+\infty} a_{g 0}^{2} p_{A_{g 0}}\left(a_{g 0}\right) d a_{g 0}=c,
\end{aligned}
$$

in which $\underline{A}_{g 0}=E\left\{A_{g 0}\right\}$ and where the second-order moment $c$ of random variable $A_{g 0}$ is expressed as $c=\underline{A}_{g 0}^{2}\left(1+\delta_{A_{g 0}}^{2}\right)$ with $\delta_{A_{g 0}}$ the coefficient of variation of $A_{g 0}$. The pdf for $Y$ is written as

$$
p_{Y}(y)=\mathbf{1}_{] 0,+\infty[}(y) \frac{1}{\underline{Y}}\left(\frac{1}{\delta_{Y}^{2}}\right)^{\frac{1}{\delta_{Y}^{2}}} \times \frac{1}{\Gamma\left(1 / \delta_{Y}^{2}\right)}\left(\frac{y}{\underline{Y}}\right)^{\frac{1}{\delta_{Y}^{2}}-1} \exp \left(-\frac{y}{\delta_{Y}^{2} \underline{Y}}\right),
$$

in which $\underline{Y}=E\{Y\}$, where $\delta_{Y}=\sigma_{Y} / \underline{Y}$ is the coefficient of variation of the random variable $Y$ such that $0 \leq \delta_{Y}<1 / \sqrt{2}$ with $\sigma_{Y}$ the standard deviation of $Y$ and where $\Gamma(\alpha)=\int_{0}^{+\infty} t^{\alpha-1} e^{-t} d t$ is the Gamma function.

In (Cataldo et al., 2009) the prior pdf of $Q$ was also constructed using the Maximum Entropy Principle and it was a Gamma distribution. The idea here is to start from an uniform distribution for $Q$ and then update the pdf using the Bayes method and experimental data. It is also important to remember that $Q$ is a tension factor and it is very difficult to be measured directly. This justifies the methodology presented here to find its corresponding pdf.

\section{Stochastic system with the prior and the posterior probabilistic models and experimental validation}

The fundamental frequency $f_{0}$ can be written as $f_{0}=\mathcal{M}\left(q, a_{g 0}, y\right)$ in which the nonlinear mapping $\left(q, a_{g 0}, y\right) \mapsto \mathcal{M}\left(q, a_{g 0}, y\right)$ is not explicitly known but is implicitly defined by solving Eqs. (1) and (2). As explained above, the stochastic system is deduced from the deterministic one substituting $q, a_{g 0}$ and $y$ by the random variables $Q, A_{g 0}$ and $Y$. Consequently, the random fundamental frequency $F_{0}$ is the random variable defined by $F_{0}=\mathcal{M}\left(Q, A_{g 0}, Y\right)$. A realization of the random fundamental frequency is calculated as the inverse of the period of the realization of the random voice signal.

\subsection{Experimental data considering a normal voice}

In order to validate the development presented here, voice signals produced by one person have been analyzed and their statistics have been compared with simulations. A voice signal corresponding to a sustained vowel /a/ has been recorded from one person and $\nu_{\exp }=1800$ frames were obtained from this signal, each one with $0.01 s$ of length. For each frame, the corresponding fundamental frequency has been calculated. The experimental data are then made up of the $\nu_{\exp }$ values $f_{0}^{\exp , 1}, \ldots, f_{0}^{\text {exp }, \nu_{\text {exp }}}$ of the fundamental frequency. So the experimental pdf of the fundamental frequency, denoted by $p_{F_{0}}^{\exp }\left(f_{0}\right)$, is estimated using the kernel density estimation method (nonparametric statistics, see for instance [?

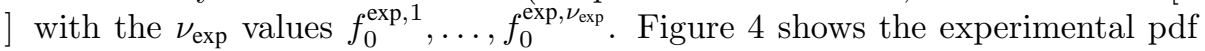
of the fundamental frequency deduced from the experimental data. 


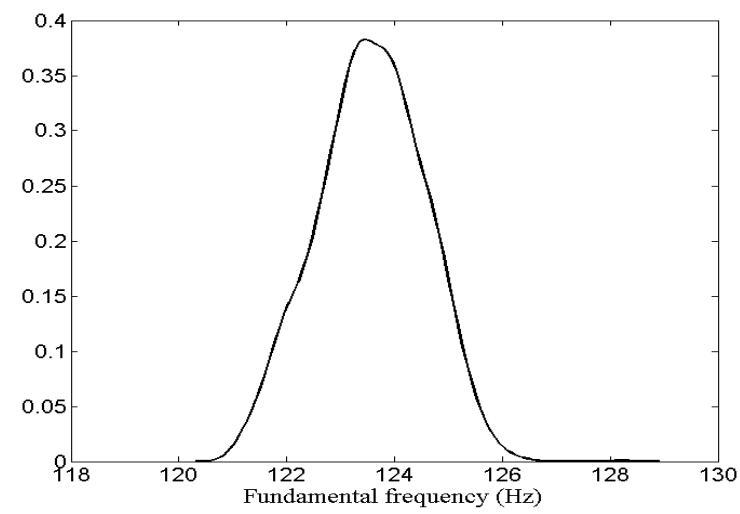

Figure 4: Experimental probability density function $f_{0} \mapsto p_{F_{0}}^{\exp }\left(f_{0}\right)$ for the fundamental frequency

\subsubsection{Posterior pdf of $Q$}

The estimation of the posterior pdf, $p_{Q}^{\text {post }}$, of the random variable $Q$ using experimental data for the fundamental frequency and the Bayes method is discussed below. The posterior pdf $p_{Q}^{\text {post }}$ is then given by the Bayes formula (Bernardo and Smith, 2000; Kaipio and Somersalo, 2005; Congdon, 2007; Carlin and Louis, 2009),

$$
p_{Q}^{\text {post }}(q)=L^{\text {bayes }}(q) p_{Q}^{\text {prior }}(q),
$$

in which $L^{\text {bayes }}(q)$ is the likelihood function defined by

$$
L^{\text {bayes }}(q)=\frac{\prod_{\ell=1}^{\nu_{\text {exp }}} p_{F_{0} \mid Q}\left(f_{0}^{\exp , \ell} \mid q\right)}{E\left\{\prod_{\ell=1}^{\nu_{\text {exp }}} p_{F_{0} \mid Q}\left(f_{0}^{\exp , \ell} \mid Q^{\text {prior }}\right)\right\}} .
$$

\subsubsection{Posterior $p d f$ of $F_{0}$}

The posterior pdf, $p_{F_{0}}^{\text {post }}$, of the random variable $F_{0}$ (fundamental frequency) is then given by

$$
p_{F_{0}}^{\text {post }}\left(f_{0}\right)=\int_{\mathbb{R}} p_{F_{0} \mid Q}\left(f_{0} \mid q\right) p_{Q}^{\text {post }}(q) d q
$$

in which $p_{F_{0} \mid Q}\left(f_{0} \mid q\right)$ is the conditional pdf of $F_{0}$, given $Q=q$. Using Eqs. (11) and (13) yields,

$$
p_{F_{0}}^{\text {post }}\left(f_{0}\right)=E\left\{L^{\text {bayes }}\left(Q^{\text {prior }}\right) p_{F_{0} \mid Q}\left(f_{0} \mid Q^{\text {prior }}\right)\right\} .
$$




\subsubsection{Computational aspects}

Let $Q^{\text {prior }}\left(\theta_{1}\right), \ldots, Q^{\text {prior }}\left(\theta_{\nu}\right)$ be $\nu=100$ independent realizations of the random variable $Q^{\text {prior }}$ for which the prior pdf is $p_{Q}^{\text {prior }}$ and with $\nu$ sufficiently large. The posterior pdf $p_{F_{0}}^{\text {post }}\left(f_{0}\right)$ of the random variable $F_{0}$ is then estimated by

$$
p_{F_{0}}^{\text {post }}\left(f_{0}\right) \simeq \frac{1}{\nu} \sum_{\ell=1}^{\nu} L^{\text {bayes }}\left(Q^{\text {prior }}\left(\theta_{\ell}\right) p_{F_{0} \mid Q}\left(f_{0} \mid Q^{\text {prior }}\left(\theta_{\ell}\right)\right) .\right.
$$

The prior pdf is defined by Eq. (5) with $a=0.65$ and $b=0.70$. This interval has been chosen in taking into account the values of the experimental data of the fundamental frequency.

Figure 5 illustrates how to obtain the conditional pdf $p_{F_{0} \mid Q}\left(f_{0} \mid Q^{\text {prior }}\left(\theta_{\ell}\right)\right)$.

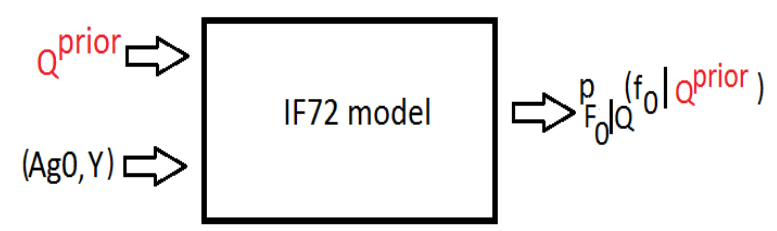

Figure 5: Generation of $p_{F_{0} \mid Q}\left(f_{0} \mid Q^{\text {prior }}\left(\theta_{\ell}\right)\right)$.

For each fixed realization $Q^{\text {prior }}\left(\theta_{\ell}\right)$ with $\ell=1, \ldots, \nu$, the conditional probability density function $p_{F_{0} \mid Q}\left(f_{0} \mid Q^{\text {prior }}\left(\theta_{\ell}\right)\right)$ is calculated with the kernel density estimation method, with $\nu^{\prime}=100$ independent realizations $F_{0}\left(\theta_{1}^{\prime} \mid \theta_{\ell}\right), \ldots, F_{0}\left(\theta_{\nu^{\prime}}^{\prime} \mid \theta_{\ell}\right)$ of random variable $F_{0}$ calculated with the stochastic model for $q=Q\left(\theta_{\ell}\right)$ and for the $\nu^{\prime}$ independent realizations $\left(A_{g 0}, Y\right)\left(\theta_{1}^{\prime}\right), \ldots,\left(A_{g 0}, Y\right)\left(\theta_{\nu^{\prime}}^{\prime}\right)$ of vector-valued random variable $\left(A_{g 0}, Y\right)$, using the prior probability model defined in Section 2. The calculations are carried out with the following values: $\underline{A}_{g 0}=0.05$, $\delta_{A_{g 0}}=0.03, \underline{Y}=750 P a$ and $\delta_{Y}=0.03$. These values were chosen according the following procedure:

Step 1: The values of $q, a_{g 0}$ and $y$ are identified to obtain the experimental value $f_{0}=123.56$ of the fundamental frequency with the deterministic model. This is the mean value of the experimental fundamental frequency. Then, a trial method, iterative, is used to identify the parameters, with a cost function constructed taking the square of the difference between fundamental frequencies (experimental and simulated). The initial values of $q, a_{g 0}$ and $y$ for solving the identification problem are considered the typical values obtained from the literature. The values obtained here were $q=0.67, a_{g 0}=5 \times 10^{-2} m^{2}$ and $y=750 P a$.

Step 2: The values of $a_{g 0}$ and $y$ found in step 1 are then used as the mean values $A_{g 0}$ and $\underline{Y}$ of the random variables $A_{g 0}$ and $Y$. Therefore, the values considered here were $m_{A_{g 0}}=5 \times 10^{-2} \mathrm{~m}^{2}$ and $m_{Y}=750 \mathrm{~Pa}$. 
Step 3: With the mean values defined in step 2, the values of $\delta_{A_{g 0}}$ and $\delta_{Y}$ are identified, with $q$ maintained fixed, to obtain the experimental value $\delta_{F_{0}}=0.008$ of the coefficient of variation of the fundamental frequency with the stochastic mechanical model. The values obtained here were $\delta_{A_{g 0}}=0.03$ and $\delta_{Y}=0.03$.

Step 4: The values of $m_{A_{g 0}}, \delta_{A_{g 0}}, m_{Y}$ and $\delta_{Y}$ are maintained and the values of $Q$ varied from 0.65 up to 0.7 , in order to obtain all the experimental frequencies, from the minimum value up to the maximum one.

Figure 6 displays the $\nu$ graphs of functions $f_{0} \mapsto p_{F_{0} \mid Q}\left(f_{0} \mid Q^{\text {prior }}\left(\theta_{\ell}\right)\right)$ for $\ell=$ $1, \ldots, \nu$ and the graph of $f_{0} \mapsto p_{F_{0}}^{\exp }\left(f_{0}\right)$. Then, for each value $q=Q\left(\theta_{\ell}\right)$,

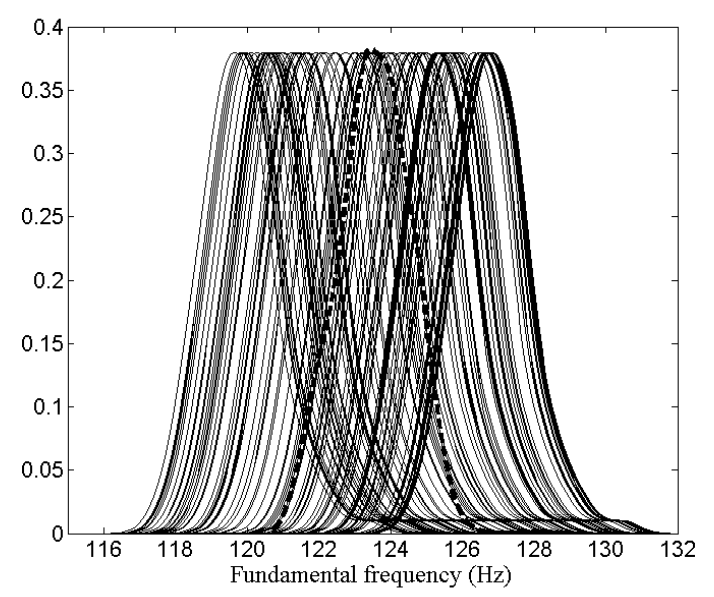

Figure 6: Graphs of conditional pdf's $f_{0} \mapsto p_{F_{0} \mid Q}\left(f_{0} \mid Q^{\text {prior }}\left(\theta_{\ell}\right)\right)$ for $\ell=1, \ldots, \nu$ (one hundred thin lines). Graph of pdf $f_{0} \mapsto p_{F_{0}}^{\exp }\left(f_{0}\right)$ of the fundamental frequency (dotted line).

$L^{\text {bayes }}(q)$ is calculated with Eq. (12) and the denominator is estimated by

$$
E\left\{\prod_{\ell=1}^{\nu_{\text {exp }}} p_{F_{0} \mid Q}\left(f_{0}^{\exp , \ell} \mid Q^{\text {prior }}\right)\right\} \simeq \frac{1}{\nu} \sum_{\ell=1}^{\nu} \prod_{\ell=1}^{\nu_{\text {exp }}} p_{F_{0} \mid Q}\left(f_{0}^{\exp , \ell} \mid Q^{\text {prior }}\left(\theta_{\ell}\right) .\right.
$$

The posterior pdf $p_{Q}^{\text {post }}(q)$ is estimated using Eq. (11) and the posterior pdf $p_{F_{0}}^{\text {post }}\left(f_{0}\right)$ of random fundamental frequency is estimated using Eq. (15). Figure 7 displays the graph of the prior pdf $q \mapsto p_{Q}^{\text {prior }}(q)$ and the graphs of posterior pdf $q \mapsto p_{Q}^{\text {post }}(q)$ for the values $\nu_{\exp }=1,100,500$ and 1800 obtained using the procedure described above (step 1 up to step 4). It should be noted that the posterior pdf of $Q$ has a narrow support centered to the value 0.675 . Then, the pdf of $Q$ was updated from experimental data. In order to validate this result, 


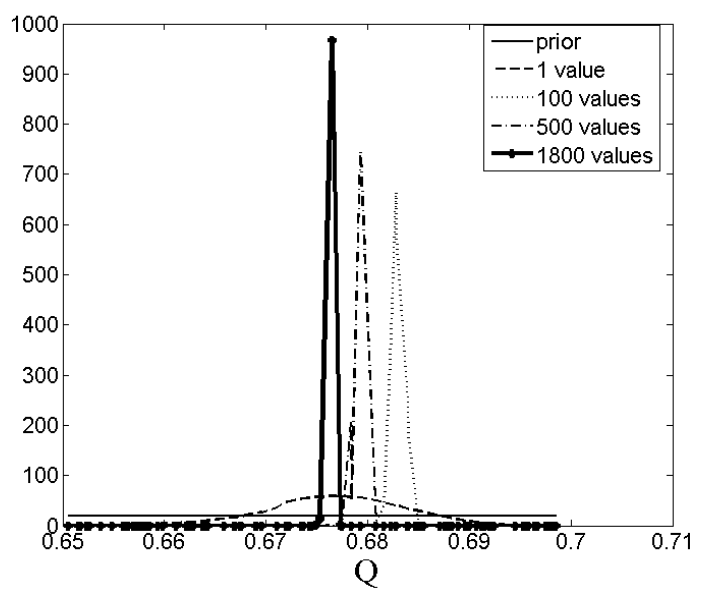

Figure 7: Graph of the prior pdf $q \mapsto p_{Q}^{\text {prior }}(q)$ and graph of the posterior pdf $q \mapsto p_{Q}^{\text {post }}(q)$ for the values $\nu_{\exp }=1,100,500$ and 1800 .

the posterior pdf of $F_{0}$ will be constructed and compared with the pdf corresponding of the experimental data. Figure 8 shows the graph of the posterior pdf $f_{0} \mapsto p_{F_{0}}^{\text {post }}\left(f_{0}\right)$ of the random fundamental frequency for the values $\nu_{\exp }=1$, 100,500 and 1800 and the graph of the experimental pdf $f_{0} \mapsto p_{F_{0}}^{\exp }\left(f_{0}\right)$. It can

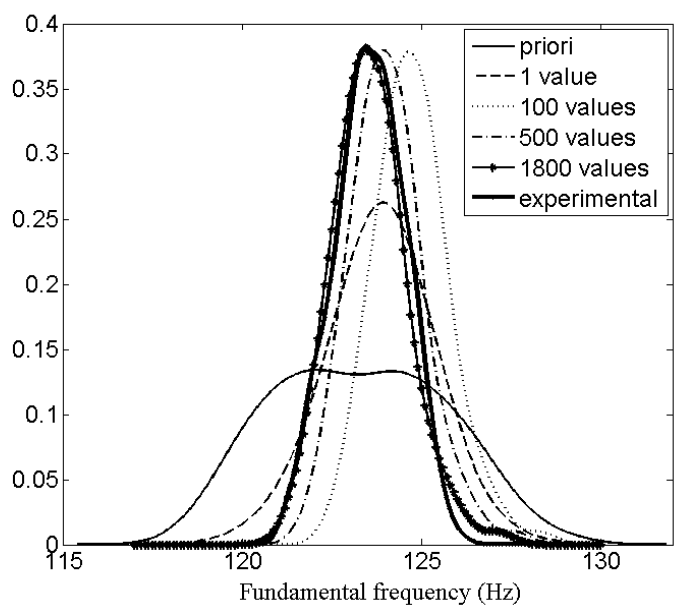

Figure 8: Graph of the posterior pdf $f_{0} \mapsto p_{F_{0}}^{\text {post }}\left(f_{0}\right)$ of the random fundamental frequency for the values $\nu_{\exp }=1,100,500$ and 1800 and graph of the experimental pdf $f_{0} \mapsto p_{F_{0}}^{\exp }\left(f_{0}\right)$. 
be noted that when 500 values are used, the posterior pdf of $F_{0}$ is very near of the experimental pdf and for 1800 values, they are almost the same, which confirms the methodology presented (see Figure 9, in which the pdfs are showed separately).

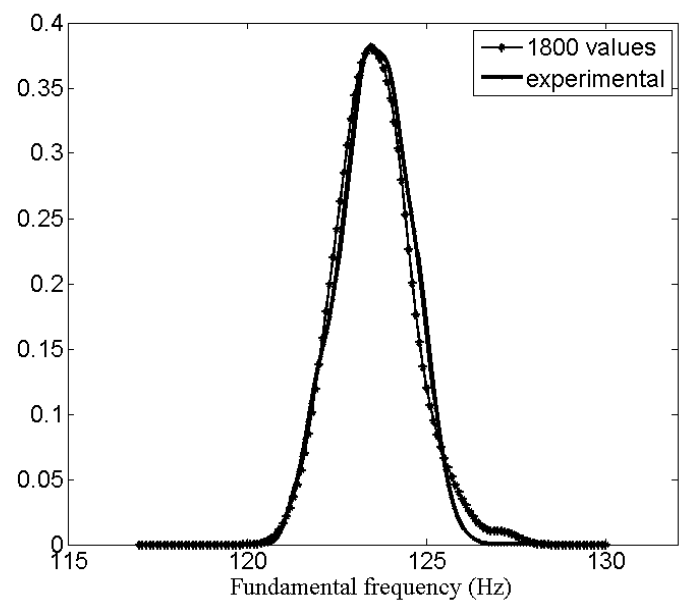

Figure 9: Graph of the posterior pdf $f_{0} \mapsto p_{F_{0}}^{\text {post }}\left(f_{0}\right)$ of the random fundamental frequency for $\nu_{\text {exp }}=1800$ and graph of the experimental pdf $f_{0} \mapsto p_{F_{0}}^{\exp }\left(f_{0}\right)$.

\subsection{Experimental data considering a case of a pathology in the vocal folds}

An application was performed considering signals from a person with a nodulus in the vocal folds. In this case, a voice signal also corresponding to a sustained vowel $/ \mathrm{a} /$ has been recorded considering $\nu_{\exp }=440$ frames, each one with $0.01 s$ of length. Figure 10 shows the probability density function obtained from the experimental values of the fundamental frequency.

It can be observed that the curve obtained has a different shape from the one obtained with a normal voice.

The procedure followed here to find the posterior pdf of $Q$ is the same used in the case of a normal voice. The values obtained from step 1 up to step 4 are described in the following:

Step 1: The values of $q, a_{g 0}$ and $y$ were identified to obtain the experimental value $f_{0}=193.02$ of the fundamental frequency with the deterministic model, here the mean value of the fundamental frequency for the pathological case. Again, a trial method, iterative, was used to identify the parameters. And also the cost function constructed takes the square of 


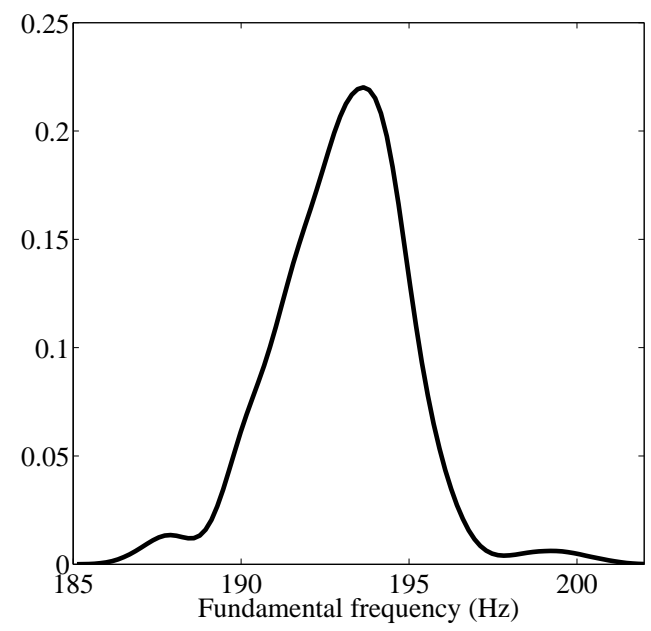

Figure 10: Experimental probability density function $f_{0} \mapsto p_{F_{0}}^{\exp }\left(f_{0}\right)$ for the fundamental frequency considering the pathological case.

the difference between fundamental frequencies (experimental and simulated). The values obtained here were $q=0.76, a_{g 0}=5 \times 10^{-2} \mathrm{~m}^{2}$ and $y=1000 P a$.

Step 2: The mean values considered for the random variables $A_{g 0}$ and $Y$ were then $m_{A_{g 0}}=5 \times 10^{-2} \mathrm{~m}^{2}$ and $m_{Y}=1000 \mathrm{~Pa}$.

Step 3: The experimental value for the coefficient of variation of the fundamental frequency was $\delta_{F_{0}}=0.01$. And the values obtained for the coefficients of variation of the other random variables were $\delta_{A_{g 0}}=0.03$ and $\delta_{Y}=0.02$.

Step 4: The values of $m_{A_{g 0}}, \delta_{A_{g 0}}, m_{Y}$ and $\delta_{Y}$ were maintained and the values of $Q$ varied from 0.750 up to 0.775 .

In this case, the prior pdf of $Q$ was constructed considering $a=0.750$ and $b=0.775$ (from step 4 ) and the same procedure as for the normal voice was used in order to find the posterior pdf of $Q$. Figure 11 shows the graph of prior pdf $q \mapsto p_{Q}^{\text {prior }}(q)$ and the graphs of posterior $\operatorname{pdf} q \mapsto p_{Q}^{\text {post }}(q)$ for $\nu_{\text {exp }}=1$, 100 and 440. The posterior pdf of $Q$ has a narrow support centered in 0.762 . It can be observed that the graphs constructed with 100 experimental values and with 440 experimental values are almost the same. In order to validate the results, Figure 12 is constructed showing the graphs of the posterior pdf $f_{0} \mapsto p_{F_{0}}^{\text {post }}\left(f_{0}\right)$ of the random fundamental frequency for $\nu_{\text {exp }}=1,100$ and 440 and the graph of the experimental pdf $f_{0} \mapsto p_{F_{0}}^{\exp }\left(f_{0}\right)$. The pdfs constructed with 440 experimental values and the corresponding pdf constructed directly from the experimental data are very near. It is important to note that there is a difference between the pdfs, which did not happen when a normal voice 


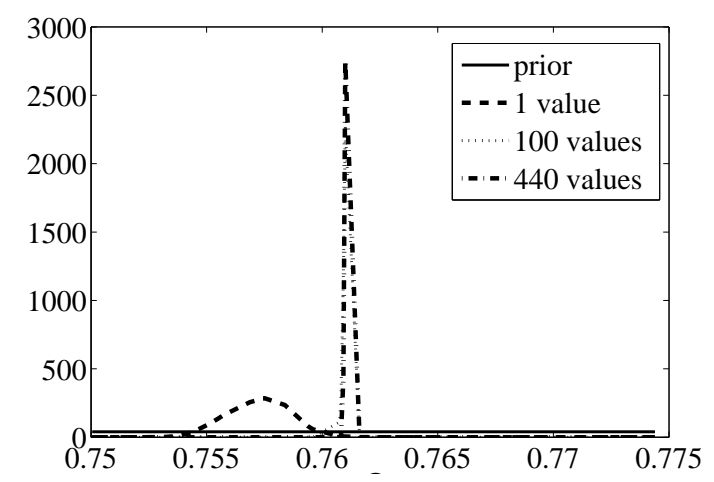

Figure 11: Graph of the prior pdf $q \mapsto p_{Q}^{\text {prior }}(q)$ and graph of the posterior pdf $q \mapsto p_{Q}^{\text {post }}(q)$ for the values of $\nu_{\exp }=1,100$ and 440 .

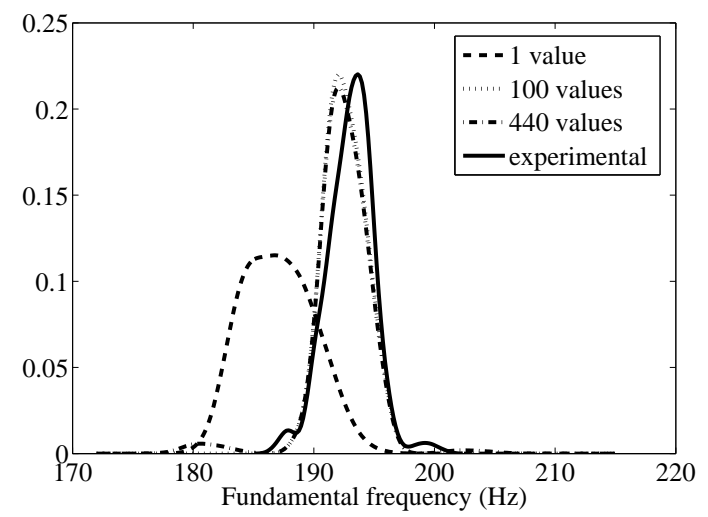

Figure 12: Graph of the posterior pdf $f_{0} \mapsto p_{F_{0}}^{\text {post }}\left(f_{0}\right)$ of the fundamental frequency for the values $\nu_{\text {exp }}=1,100$ and 440 and graph of the experimental pdf $f_{0} \mapsto p_{F_{0}}^{\exp }\left(f_{0}\right)$.

was considered. However, one can say that the methodology discussed here is very well applied because in the case of a pathology the pdf constructed from experimental data has really a particular shape.

\section{Conclusions}

A methodology to perform the experimental identification of an uncertain mechanical model for producing voice is proposed. Such a model is mainly sensitive to three system parameters, and in particular, to the tension parameter which controls the fundamental frequency of the vocal-fold vibrations. This methodology developed to solve a statistical inverse problem consists in constructing the prior stochastic models of the three parameters and then to use 
the Bayes method to construct the posterior stochastic model of the random tension parameter (which cannot be measured directly) using the observable quantity of the system which is the fundamental frequency. The likelihood function is constructed using the system considered to produce voice. The posterior stochastic model of the random fundamental frequency has been deduced and all the useful numerical formula which allows the methodology to be implemented for the calculations has been presented. Two experimental validations have been presented using experimental data, one considering a normal voice and another considering a case with pathology in the vocal folds. The methodology applied showed to be efficient in both cases. The validated posterior stochastic mechanical model then allows voice signals to be simulated.

\section{ACKNOWLEDGEMENTS}

This work was supported by Fundação de Amparo à Pesquisa no Rio de Janeiro (FAPERJ - programa Jovens Cientista do Nosso Estado), by CAPES (CAPES/COFECUB project N. 672/10) and by the Brazilian Agency Conselho Nacional de Desenvolvimento Científico e Tecnológico (CNPq).

\section{REFERENCES}

Bernardo, J. M., Smith, A. F. M., 2000. Bayesian Theory. John Wiley \& Sons, Chichester.

Bowman, A.W., Azzalini A., 1997. Applied Smoothing Techniques for Data Analysis, Oxford University Press.

Carlin, B.P., Louis T.A., 2009. Bayesian Methods for Data Analysis, Third Edition, CRC Press, Boca Raton.

Cataldo, E., Sampaio R., Lucero, J., Soize, C., 2008. Modeling random uncertainties in voice production using a parametric approach. Mechanics Research Communication 35, 429-490.

Cataldo, E., Soize C., Sampaio R., Desceliers, C., 2009. Probabilistic modeling of a nonlinear dynamical system used for producing voice. Computational Mechanics 43, 265-275.

Congdon P., 2007. Bayesian Statistical Modelling, Second Edition. John Wiley \& Sons, Chichester.

Ishizaka, K., Flanagan, J., 1972. Synthesis of voiced sounds from a two-mass model of the vocal folds. Bell Syst. Tech. J. 51,1233-1268.

Jaynes, E., 1957a. Information theory and statistical mechanics. Phys. Rev. 106(4),620-630.

Jaynes, E., 1957b. Information theory and statistical mechanics. Phys. Rev. 108,171-190.

Kaipio J., Somersalo E., 2005. Statistical and Computational Inverse Problems. Springer-Verlag, New York. 
Koizumi, T., Taniguchi, S., Hiromitsu, S., 1976. Two-mass models of the vocal folds for natural sounding voice synthesis. J. Acoust. Soc. Am. $82,1179-1183$.

Lous, N. J. C., Hofmans, G. C. J., Veldhuis, R. N. J., Hirschberg, A., 1998. Symmetrical two-mass vocal-fold model coupled to vocal tract and trachea with application to prosthesis design. Acta Acustica 84(6), 1135-1150.

Shannon, C. E., 1948. A mathematical theory of communication. Bell System Tech. 28, 379-423 and 623-659.

Titze, I., 1994. Principles of voice production. Prentice Hall, Englewood Cliffs, NJ.

Zhang, Y., Jiang, J., Rahn III, D. A., 2005. Studying vocal fold vibrations in Parkinson's disease with a nonlinear model. Chaos 15,1-10. 